\title{
A new discretization scheme for one dimensional stochastic differential equations using time change method
}

\author{
Masaaki Fukasawa* $\quad$ Mitsumasa Ikeda $^{\dagger}$
}

\begin{abstract}
We propose a new numerical method for one dimensional stochastic differential equations (SDEs). The main idea of this method is based on a representation of a weak solution of an SDE using a time-changed Brownian motion, which dates back to Doeblin (1940). In cases where the diffusion coefficient is bounded and is $\beta$-Hölder continuous with $0<\beta \leq 1$, we provide the rate of strong convergence. An advantage of our approach is that we approximate the weak solution, which enables us to treat SDEs with no strong solution. Our scheme is the first to achieve strong convergence for the case of $0<\beta<1 / 2$.
\end{abstract}

Keywords: stochastic differential equations; numerical analysis.

MSC2020 subject classifications: $65 \mathrm{C} 30 ; 91 \mathrm{G} 60$; $60 \mathrm{H} 35$.

Submitted to ECP on May 26, 2020, final version accepted on July 16, 2021.

\section{Introduction}

In this article, we provide a numerical method for approximating a weak solution of a one dimensional stochastic differential equation. There are many studies on numerical approximation of SDEs which converge strongly to the solution. There are a variety of applications, including path-dependent option pricing in financial engineering. In this work, we focus on the following one-dimensional SDE:

$$
d X_{t}=\sigma\left(t, X_{t}\right) d W_{t} .
$$

This kind of SDE model is called a local volatility model and is popular in financial practice. Although (1.1) does not include a drift term, we note that under appropriate conditions, a general one-dimensional SDE with drift can be reduced to (1.1). Time homogeneous one-dimensional SDEs can be transformed to not have a drift term by using a scale function in the pathwise sense, and time inhomogeneous SDEs can also be transformed to (1.1) by using the Girsanov-Maruyama transformation in the sense of law.

In order to study numerical schemes of the SDE (1.1), we need to discuss the conditions under which the existence and uniqueness of the solution hold in various

\footnotetext{
*Graduate School of Engineering Science, Osaka University, 1-3, Machikaneyama-cho, Toyonaka, Osaka, 560-8531, Japan, E-mail: fukasawa@sigmath.es . osaka-u.ac.jp

${ }^{\dagger}$ Graduate School of Engineering Science, Osaka University, 1-3, Machikaneyama-cho, Toyonaka, Osaka, 560-8531, Japan, E-mail: mikeda@sigmath.es.osaka-u.ac.jp
} 
different senses: strong uniqueness, pathwise uniqueness, and uniqueness in the sense of probability law. Many researchers have studied the unique existence of the solution to SDEs for a long time. The most famous condition for strong unique existence of a solution is the Lipschitz continuity of the drift and diffusion coefficients (see [11]).

According to Bru and Yor [3], W. Doeblin wrote a paper about this issue before many facts about the structure of martingale were found. He showed that a diffusion process can be represented by some stochastic process driven by a time-changed Brownian motion. Although this work of Doeblin from 1940 was only made public in 2000, the idea was rediscovered and extended in stochastic calculus, and was already in a textbook [10] by Ikeda and Watanabe in 1984, where it was shown that a certain class of one-dimensional SDE of the form (1.1) has a unique solution represented by a time changed Brownian motion, where the time change is given as the solution of a random ordinary differential equation, as we discuss in the next section in more detail. We use this representation to construct a new approximation scheme for one-dimensional SDEs. For the time homogeneous case, namley, $\sigma(t, x)=\sigma(x)$ in (1.1), Engelbert and Schmidt [4] gave an an equivalent condition for weak existence and uniqueness in the sense of probability law, under which the weak solution is represented by a time-changed Brownian motion. For time-homogeneous SDEs, an excellent survey [14] about the existence and uniqueness of SDEs is available.

The most famous numerical scheme for SDEs is the Euler-Maruyama scheme. This method approximates a solution of an SDE in a very similar way to the Euler scheme for ordinary differential equations. We define the Euler-Maruyama approximations of $X_{t}, t \in[0, T)$ as the solutions of

$$
d X_{t}^{(n)}=\sigma\left(t, X_{\frac{\lfloor n t\rfloor}{n}}^{(n)}\right) d W_{t},
$$

where $X_{0}$ is a given initial value. It is well known that the Euler-Maruyama approximation converges to the strong solution of a corresponding SDE uniformly in the sense of $L^{p}$ with convergence rate $n^{-1 / 2}$ when the diffusion coefficient is Lipschitz continuous [12]. Under $\beta$-Hölder continuity of the diffusion coefficient $\sigma(t, x)$, where $1 / 2 \leq \beta \leq 1$, Gyöngy and Rásonyi [7] showed that for any $T>0$ there exists a constant $C>0$ such that

$$
\left\{E\left[\sup _{0 \leq t \leq T}\left|X_{t}-X_{t}^{(n)}\right|^{p}\right]\right\}^{1 / p} \leq \begin{cases}\left(\frac{C}{\ln n}\right)^{1 / 2 p} & \text { if } \beta=1 / 2 \\ C n^{-(\beta-1 / 2) / p} & \text { if } \beta \in(1 / 2,1) \\ C n^{-(\beta / 2-1 / 4)} & \text { if } \beta=1\end{cases}
$$

for any $n \geq 2$ and $p \geq 2$, where $X_{t}$ is the strong solution of the $\operatorname{SDE}(1.1)$ and $X_{t}^{(n)}$ is the corresponding Euler-Maruyama approximation for step size $1 / n$.

When $\beta<1 / 2$, a strong solution does not exist in general [1] and no numerical schemes have been proposed. Note that this kind of rough diffusion coefficient appears when we deal with random medium. For example, Brox considered in [2] a one-dimensional diffusion process in which the drift coefficient is an independent white noise. As discussed in [2] and [9], we can remove the distributional drift coefficient by scale transformation and obtain an SDE of the form (1.1) with $\sigma(t, x)=e^{B\left(s^{-1}(x)\right)}$, where $B$ is an independent two-sided Brownian motion and $s$ is the scale function.

In Section 2, we propose a new method of approximating the SDE (1.1). In Section 3, we provide the convergence rates of our method under the $\beta$-Hölder condition with $0<\beta \leq 1$ or under a certain smoothness condition. One advantage of our approach is that we approximate the weak solution, which enables us to treat an SDE that does not have a strong solution. Our scheme is the first to achieve strong convergence for 
$0<\beta<1 / 2$, and provides a better convergence rate than in [7] for $1 / 2 \leq \beta<2 / 3$. In Section 4, we provide some numerical experiments on one-dimensional SDEs with a Hölder continuous diffusion coefficient.

\section{Discretization with time change}

Let $\left(\Omega, \mathcal{F}, \mathbb{P},\left\{\mathcal{F}_{t}\right\}_{t \geq 0}\right)$ be a filtered probability space. We consider a new discretization scheme for the one-dimensional SDE (1.1) on this space. Our method is based on the following theorem from [10].

Theorem 2.1 (Preliminary theorem). Let $\left\{b_{t}\right\}$ be a one-dimensional $\left\{\mathcal{F}_{t}\right\}$-Brownian motion with $b_{0}=0$ on $\left(\Omega, \mathcal{F}, \mathbb{P},\left\{\mathcal{F}_{t}\right\}_{t \geq 0}\right)$ and let $X_{0}$ be an $\mathcal{F}_{0}$-measurable random variable. Define a continuous process $\left\{\xi_{t}\right\}$ by $\xi_{t}=X_{0}+b_{t}$. Let $\{\varphi(t)\}$ be a.s. a solution of the ODE

$$
\varphi(t)=\int_{0}^{t} \frac{\mathrm{d} s}{\sigma^{2}\left(\varphi(s), \xi_{s}\right)}
$$

If we then define $X_{t}=\xi_{\varphi^{-1}(t)}=X_{0}+b_{\varphi^{-1}(t)}$ and $\tilde{\mathcal{F}}_{t}=\mathcal{F}_{\varphi^{-1}(t)}$, there exists an $\left\{\tilde{\mathcal{F}}_{t}\right\}$ Brownian motion $\left\{W_{t}\right\}$ such that $\left(\left\{X_{t}\right\},\left\{W_{t}\right\}\right)$ is a weak solution of (1.1) on the probability space $(\Omega, \mathcal{F}, \mathbb{P})$ with filtration $\left\{\tilde{\mathcal{F}}_{t}\right\}$. Moreover, if the solution of the ODE (2.1) is unique a.s., then the solution of (1.1) is unique in law.

Remark 2.2. A sufficient condition for the ODE (2.1) to be well-posed for a fixed $\omega \in \Omega$, is that $\sigma\left(y, \xi_{t}(\omega)\right)$ is locally Lipschitz continuous in $y$ and satisfies the inequality

$$
\left|\sigma^{-2}\left(y, \xi_{t}(\omega)\right)\right| \leq a(t)|y|+b(t)
$$

for all $t \in[0, \infty)$ and $y \in \mathbb{R}$, where $a(t)$ and $b(t)$ are some continuous non-negative (and possibly random) functions of $t$ (refer to [6]). In the next section, we will show the convergence rate of our method, where we assume boundedness of the diffusion coefficient (see Condition 3.1). Then, it is easy to verify that the local Lipschitz continuity of $\sigma\left(y, \xi_{t}(\omega)\right)$ in $y$ is sufficient because the condition (2.2) follows from the boundedness of $\sigma^{-2}\left(y, \xi_{t}(\omega)\right)$.

The main goal of this paper is to build a numerical approximation of a solution $\left\{X_{t}\right\}$ of the SDE (1.1) using Theorem 2.1. In order to approximate this time-changed Brownian motion, we first make an approximation of Brownian motion $\left\{\xi_{t}\right\}$ by $\left\{\xi_{t}^{(n)}\right\}$ that is a linear interpolation of a random walk generated by normal distributed random variables, that is,

$$
\xi_{t}^{(n)}:=\xi_{\lfloor n t\rfloor / n}+\left(t-\frac{\lfloor n t\rfloor}{n}\right)\left(\xi_{(\lfloor n t\rfloor+1) / n}-\xi_{\lfloor n t\rfloor / n}\right)
$$

where $\left(\xi_{(\lfloor n t\rfloor+1) / n}-\xi_{\lfloor n t\rfloor / n}\right) \sim \mathcal{N}(0,1 / n)$. Second, we approximate $\{\varphi(t)\}$ by $\left\{\varphi_{n}(t)\right\}$, the Euler method for ordinary differential equation, namely,

$$
\begin{aligned}
& \varphi_{n}(0)=\varphi(0)=0, \\
& \varphi_{n}(t)=\varphi_{n}\left(\frac{k}{n}\right)+\left(t-\frac{k}{n}\right) \frac{1}{\sigma^{2}\left(\varphi_{n}(k / n), \xi_{k / n}\right)}, \quad t \in\left(\frac{k}{n}, \frac{k+1}{n}\right]
\end{aligned}
$$

Third, we make the inverse function $\tau_{n}(t)$ of $t \longmapsto \varphi_{n}(t)$ by

$$
\tau_{n}(t)=\frac{k}{n}+\frac{t-\varphi_{n}\left(\frac{k}{n}\right)}{\varphi_{n}\left(\frac{k+1}{n}\right)-\varphi_{n}\left(\frac{k}{n}\right)} \frac{1}{n}
$$

where $t \in\left[\varphi_{n}\left(\frac{k}{n}\right), \varphi_{n}\left(\frac{k+1}{n}\right)\right)$. We can easily check that $\tau_{n}(t)$ is the inverse function of $\varphi_{n}(t)$ by its definition.

Let $t_{j}, j=0,1,2, \cdots$ be defined by $t_{j}=j / n$. The full algorithm for this method is as follows. 
STEP1 Construct $\xi_{t_{j}}, j=0,1,2, \cdots$ using a normal distributed random sequence $\left\{\xi_{k}-\right.$ $\left.\xi_{k-1}\right\}_{k=1, \cdots, j}$ with $\xi_{0}=X_{0}$ and compute the $\varphi_{n}\left(t_{j}\right)$ for each $j$. As we prove later, under Condition $3.1, \varphi_{n}(t)$ is strictly increasing and $\varphi\left(t_{j}\right)$ goes to infinity as $j \rightarrow \infty$. This makes the next step valid.

STEP2 The first time $\varphi_{n}\left(t_{j}\right)$ crosses $t$ (i.e., at the first step $j$ such that $\varphi_{n}\left(t_{j}\right)>t$ ), calculate $\tau_{n}(t)$ using the formula (2.6) where we select $k$ in (2.6) to be $k=j-1$.

STEP3 Using $\tau_{n}(t)$ and (2.3), calculate $\xi_{\tau_{n}(t)}^{(n)}$ i.e.,

$$
\xi_{\tau_{n}(t)}^{(n)}=\xi_{t_{j-1}}+\left(\tau_{n}(t)-t_{j-1}\right)\left(\xi_{t_{j}}-\xi_{t_{j-1}}\right) .
$$

We thus obtain a path of $\xi_{\tau_{n}(t)}^{(n)}$ using [STEP1]-[STEP3]. The main result of this paper is the discretization error of $\left\{\xi_{\tau_{n}(t)}^{(n)}\right\}$ in the sense of $L^{p}$ under the Hölder condition of $\sigma(t, x)$, which is given in the next section.

\section{Rate of convergence}

In this section, we show the convergence rates of our approximation scheme. In the rest of this paper, we assume the following condition.

Condition 3.1. There are positive constants $C_{1}, C_{2}$ such that $C_{1} \leq \sigma(t, x) \leq C_{2}$ for all $(t, x) \in[0, \infty) \times \mathbb{R}$.

Theorem 3.2 states that under the $\beta$-Hölder continuity of $\sigma(t, x)$ with $\beta \in(0,1]$ our numerical approximation converges to the exact solution in the sense of $L^{p}$ uniformly, and the convergence rate is $n^{-\alpha^{2} \beta}$, where $\alpha$ is an arbitrary value smaller than $1 / 2$. Theorem 3.8 provides a more precise convergence rate $n^{-\alpha}$ when $\sigma$ is sufficiently smooth.

Theorem 3.2. Let $T>0$. Suppose that $\sigma(t, x)$ satisfies Condition 3.1 and that there exist constants $\beta \in(0,1], C_{\beta}>0$, and $L_{T}>0$ such that for $s, t \leq T$,

$$
|\sigma(s, x)-\sigma(t, y)| \leq L_{T}|s-t|+C_{\beta}|x-y|^{\beta} .
$$

Let $\xi_{t}, \xi_{t}^{(n)}, \tau(t), \tau_{n}(t)$ be defined as in the previous section. Then, for any $p \geq 1$ and $\alpha \in[0,1 / 2)$, there exists a positive constant $\tilde{K}_{T}$ such that

$$
\left\{E\left[\sup _{t \leq T}\left|\xi_{\tau_{n}(t)}^{(n)}-\xi_{\tau(t)}\right|^{p}\right]\right\}^{1 / p} \leq \tilde{K}_{T} n^{-\alpha^{2} \beta}
$$

Remark 3.3. The conditions in the theorem above are sufficient to guarantee (2.2), which means that a solution to the differential equation (2.1) uniquely exists.

We use the following lemma that is an immediate consequence of Theorem (2.1) in [13].

Lemma 3.4. Let $\left\{\xi_{t}\right\}$ be Brownian motion and denote

$$
H_{\alpha, T}:=\sup _{\substack{s \neq t \\ s, t \leq T}} \frac{\left|\xi_{t}-\xi_{s}\right|}{|t-s|^{\alpha}} .
$$

Then the function $T \mapsto H_{\alpha, T}$ is increasing and

$$
E\left[\left(H_{\alpha, T}\right)^{\gamma}\right]<\infty
$$

for any $\alpha \in[0,1 / 2)$ and $\gamma>0$. 
Lemma 3.5. Suppose that $\sigma(t, x)$ satisfies Condition 3.1 and that $\varphi(t)$ satisfies (2.1). Let $\varphi_{n}(t)$ be defined as (2.5). Then $\varphi, \varphi_{n}, \tau, \tau_{n}$ is continuous and strictly increasing. Furthermore, for each $\gamma>0$ and $T>0$ the following holds

$$
\sup _{t \leq T}\left|\tau_{n}(t)-\tau(t)\right| \leq C_{2}^{2} \sup _{t \leq C_{2}^{2} T}\left|\varphi_{n}(t)-\varphi(t)\right|
$$

Proof. It follows from Condition 3.1 and equation (2.1) that $\varphi$ and $\varphi_{n}$ are continuous and strictly increasing. Furthermore, it follows that

$$
\begin{aligned}
\varphi(t) & \geq \int_{0}^{t} \frac{d s}{C_{2}^{2}}=C_{2}^{-2} t \\
\varphi_{n}(t) & \geq \sum_{j=0}^{k-1} \frac{1}{C_{2}^{2}} \frac{1}{n}+C_{2}^{-2}\left(t-\frac{k}{n}\right)=C_{2}^{-2} t
\end{aligned}
$$

for $t \in\left[\frac{k}{n}, \frac{k+1}{n}\right)$. Therefore $\varphi(t), \varphi_{n}(t) \rightarrow \infty$ as $t \rightarrow \infty$, which implies the existence, continuity, and strictly increasing property of $\tau$ and $\tau_{n}$. It also follows that $\tau(t), \tau_{n}(t) \rightarrow \infty$ as $t \rightarrow \infty$. Because of these properties, $\varphi_{n}(t)$ is a bijection. Then by (3.5), for any $t \leq T$, there exists $t^{\prime} \leq C_{2}^{2} T$ such that $t=\varphi_{n}(t)$, and therefore

$$
\sup _{t \leq T}\left|\varphi_{n}^{-1}(t)-\varphi^{-1}(t)\right| \leq \sup _{t \leq C_{2}^{2} T} \mid\left(\varphi_{n}^{-1}\left(\varphi_{n}(t)\right)-\varphi^{-1}\left(\varphi_{n}(t)\right)\left|=\sup _{t \leq C_{2}^{2} T}\right| t-\varphi^{-1}\left(\varphi_{n}(t)\right) \mid .\right.
$$

Because of Condition 3.1 and (2.1), we obtain

$$
|\varphi(\tilde{s})-\varphi(\tilde{t})| \geq C_{2}^{-2}|\tilde{s}-\tilde{t}|,
$$

for every $\tilde{s}, \tilde{t}$. By taking $\tilde{s}=\varphi^{-1}(s), \tilde{t}=\varphi^{-1}(t)$, we get $C_{2}^{-2}$-Lipschitz continuity of $\varphi^{-1}$. Therefore, (3.6) with the Lipschitz continuity of $\varphi^{-1}$ implies

$$
\sup _{t \leq C_{2}^{2} T}\left|t-\varphi^{-1}\left(\varphi_{n}(t)\right)\right|=\sup _{t \leq C_{2}^{2} T}\left|\varphi^{-1}(\varphi(t))-\varphi^{-1}\left(\varphi_{n}(t)\right)\right| \leq C_{2}^{2} \sup _{t \leq C_{2}^{2} T}\left|\varphi(t)-\varphi_{n}(t)\right| .
$$

Thus we obtain the assertion of the lemma using the last inequality and (3.6).

Proof of Theorem 3.2. First, from Minkowski's inequality, we have

$$
\begin{aligned}
& \left\{\mathbb{E}\left[\sup _{t \leq T}\left|\xi_{\tau_{n}(t)}^{(n)}-\xi_{\tau(t)}\right|^{p}\right]\right\}^{1 / p} \\
\leq & \left\{\mathbb{E}\left[\sup _{t \leq T}\left|\xi_{\tau_{n}(t)}^{(n)}-\xi_{\frac{\left\lfloor n \tau_{n}(t)\right\rfloor}{n}}\right|^{p}\right]\right\}^{1 / p}+\left\{\mathbb{E}\left[\sup _{t \leq T}\left|\xi_{\frac{\left\lfloor n \tau_{n}(t)\right\rfloor}{n}}-\xi_{\tau(t)}\right|^{p}\right]\right\}^{1 / p},
\end{aligned}
$$

where $\lfloor t\rfloor$ is the largest integer less than $t$. Since $\xi_{t}^{(n)}$ is the interpolation of the sequence $\left\{\xi_{j / n}\right\}_{j=0,1,2, \cdots, \text { it follows that }}$

$$
\left|\xi_{t}^{(n)}-\xi_{\frac{\lfloor n t\rfloor}{n}}\right| \leq\left|\xi_{\frac{\lfloor n t\rfloor+1}{n}}^{(n)}-\xi_{\frac{\lfloor n t\rfloor}{n}}\right|=\left|\xi_{\frac{\lfloor n t\rfloor+1}{n}}-\xi_{\frac{\lfloor n t\rfloor}{n}}\right|
$$


Therefore, using Minkowski's inequality again, we obtain

$$
\begin{aligned}
&\left\{E\left[\sup _{t \leq T}\left|\xi_{\tau_{n}(t)}^{(n)}-\xi_{\tau(t)}\right|^{p}\right]\right\}^{1 / p} \\
& \leq\left\{E \left[\sup _{t \leq T} \mid \xi_{\frac{\left\lfloor n \tau_{n}(t)\right\rfloor+1}{n}}-\xi_{\left.\left.\left.\frac{\left\lfloor n \tau_{n}(t)\right\rfloor}{n}\right|^{p}\right]\right\}^{1 / p}}\right.\right. \\
&+\left\{E\left[\sup _{t \leq T}\left|\xi_{\frac{\left\lfloor n \tau_{n}(t)\right\rfloor}{n}}-\xi_{\tau_{n}(t)}\right|^{p}\right]\right\}^{1 / p} \\
&+\left\{E\left[\sup _{t \leq T}\left|\xi_{\tau_{n}(t)}-\xi_{\tau(t)}\right|^{p}\right]\right\}^{1 / p} .
\end{aligned}
$$

Let us provide the desired conclusion by estimating the convergence rate of (3.7)-(3.9) as $n \rightarrow \infty$. Define $H_{\alpha, T}$ as (3.3) for $(\alpha, T) \in(0,1 / 2) \times[0, \infty)$ and set $T^{\prime}:=\max \left\{T, C_{2}^{2} T+\right.$ $1 / n\}, \tilde{H}:=H_{\alpha, T^{\prime}}\left(\geq H_{\alpha, T}\right)$. Because $H_{t}$ is monotonically increasing with respect to $t$, Lemma 3.4 implies

$$
\begin{array}{r}
\left\{E\left[\sup _{t \leq T}\left|\xi_{\left(\left\lfloor n \tau_{n}(t)\right\rfloor+1\right) n^{-1}}-\xi_{\left\lfloor n \tau_{n}(t)\right\rfloor n^{-1}}\right|^{p}\right]\right\}^{1 / p} \leq\left\{E\left[\tilde{H}^{p}\right]\right\}^{1 / p} n^{-\alpha} \\
\left\{E\left[\sup _{t \leq T}\left|\xi_{\left\lfloor n \tau_{n}(t)\right\rfloor n^{-1}}-\xi_{\tau_{n}(t)}\right|^{p}\right]\right\}^{1 / p} \leq\left\{E\left[\tilde{H}^{p}\right]\right\}^{1 / p} n^{-\alpha}
\end{array}
$$

This gives us the rate of convergence of the terms (3.7) and (3.8). It remains to prove that the convergence rate of the term (3.9) is $n^{-\alpha^{2} \beta}$. From Lemma 3.4, Lemma 3.5 and Hölder's inequality, we have

$$
\begin{aligned}
\left\{E\left[\sup _{t \leq T}\left|\xi_{\varphi_{n}^{-1}(t)}-\xi_{\varphi^{-1}(t)}\right|^{p}\right]\right\}^{1 / p} & \leq\left\{E\left[\tilde{H}^{p} C_{2}^{2 p \alpha} \sup _{t \leq C_{2}^{2} T}\left|\varphi(t)-\varphi_{n}(t)\right|^{p \alpha}\right]\right\}^{1 / p} \\
& \leq\left\{E\left[\tilde{H}^{2 p}\right]\right\}^{1 / 2 p} C_{2}^{2 \alpha}\left\{E\left[\sup _{t \leq T^{\prime}}\left|\varphi(t)-\varphi_{n}(t)\right|^{2 p}\right]\right\}^{1 / 2 p} .
\end{aligned}
$$

We obtain the convergence rate of (3.12) by estimating the error function $e_{n}(t):=$ $\varphi_{n}(t)-\varphi(t)$. For a positive number $h$, define a function $\psi_{h}:[0, \infty) \rightarrow \mathbb{R}$ as

$$
\psi_{h}(t):=\frac{1}{h} \int_{t}^{t+h}\left(\sigma^{-2}\left(\varphi(s), \xi_{s}\right)-\sigma^{-2}\left(\varphi(t), \xi_{t}\right)\right) d s .
$$

From Lemma 3.4, condition (3.1) and the fact that $\left|a^{-2}-b^{-2}\right|=\left|a^{-1}+b^{-1}\right|\left|a^{-1} b^{-1}\right||a-b|$ for $t \leq T^{\prime}-h$, we obtain

$$
\begin{aligned}
\left|\psi_{h}(t)\right| & \leq \frac{1}{h}\left|\int_{t}^{t+h}\left\{\sigma^{-2}\left(\varphi(s), \xi_{s}\right)-\sigma^{-2}\left(\varphi(t), \xi_{t}\right)\right\} d s\right| \\
& \leq \frac{1}{h} \int_{t}^{t+h} 2 C_{1}^{-3} L_{T^{\prime}}|\varphi(s)-\varphi(t)|+C_{\beta}\left|\xi_{t}-\xi_{s}\right|^{\beta} d s \\
& \leq \frac{1}{h} \int_{t}^{t+h} 2 C_{1}^{-3}\left(L_{T^{\prime}}+C_{\beta}\right)\left|\int_{t}^{s}\right| C_{1}^{-2}|d u+| \xi_{t}-\left.\xi_{s}\right|^{\beta} \mid d s \\
& \leq \frac{1}{h} \int_{t}^{t+h} 2 C_{1}^{-3}\left(L_{T^{\prime}}+C_{\beta}\right)\left|\int_{t}^{s}\right| C_{1}^{-2}|d u+\tilde{H}| t-\left.s\right|^{\alpha \beta} \mid d s .
\end{aligned}
$$


From Lemma 3.4, there is a random variable $R$ depending on $T^{\prime}$ that has moments of all orders and satisfies the following

$$
\left|\psi_{h}(t)\right| \leq \frac{1}{h} \int_{t}^{t+h} R h^{\alpha \beta} d s=R h^{\alpha \beta} .
$$

However, from the definition of $\varphi(t)$,

$$
\varphi(t)=\varphi(s)+h \sigma^{-2}\left(\varphi(s), \xi_{s}\right)+h \psi_{h}(s) \text { for } t>s,
$$

where $h=t-s$. Then for $t \in\left(t_{i}, t_{i+1}\right]$,

$$
e_{n}(t)=e_{n}\left(t_{i}\right)+\left(t-t_{i}\right)\left\{\sigma^{-2}\left(\varphi^{(n)}\left(t_{i}\right), \xi_{t_{i}}\right)-\sigma^{-2}\left(\varphi\left(t_{i}\right), \xi_{t_{i}}\right)\right\}+\left(t-t_{i}\right) \psi_{t-t_{i}}\left(t_{i}\right),
$$

and by the Lipschitz continuity of $\sigma(t, x)$ with respect to $t$,

$$
\begin{aligned}
\left|e_{n}(t)\right| & \leq\left|e_{n}\left(t_{i}\right)\right|+\left(t-t_{i}\right) L_{T^{\prime}}\left|e_{n}\left(t_{i}\right)\right|+\left(t-t_{i}\right)\left|\psi_{t-t_{i}}\left(t_{i}\right)\right| \\
& \leq\left(1+h L_{T^{\prime}}\right)\left|e_{n}\left(t_{i}\right)\right|+R h^{\alpha \beta+1} .
\end{aligned}
$$

We repeat this calculation for $i$ and then by using the standard result for a geometric series and the fact that $1+L_{T^{\prime}} h<\mathrm{e}^{L_{T^{\prime}}} h$, we have

$$
\begin{aligned}
& \sup _{s \leq t}\left|e_{n}(s)\right| \\
\leq & \left|e_{n}\left(t_{i+1}\right)\right| \leq \sum_{j=0}^{i}\left(1+h L_{t^{\prime}}\right)^{j} R h^{\alpha \beta+1}=\frac{R h^{\alpha \beta}}{L_{T^{\prime}}}\left\{\left(1+h L_{T^{\prime}}\right)^{i+1}-1\right\} \leq \frac{R h^{\alpha \beta}}{L_{T^{\prime}}}\left\{\mathrm{e}^{L_{T^{\prime}}\left(T^{\prime}+1\right)}-1\right\} .
\end{aligned}
$$

Because of the fact that the inequality (3.13) holds for $t \leq T^{\prime}$, the integrable property of the random variable $R$, and the Cauchy-Schwartz's inequality, there exists a positive number $K$ depending on $T^{\prime}$ such that

$$
\left\{E\left[\sup _{t \leq T^{\prime}}\left|\varphi(t)-\varphi_{n}(t)\right|^{2 p \alpha}\right]\right\}^{1 / 2 p} \leq K\left(\frac{1}{n}\right)^{\alpha^{2} \beta} .
$$

This completes the proof.

Remark 3.6. We have already seen that our approximation converges to the solution of (1.1) and the rate of convergence is $n^{-\alpha^{2} \beta}$. Let us compare our result with (1.3) by Gyöngy and Rásonyi [7] which provide the rate of strong convergence of the EulerMaruyama scheme under similar conditions to ours. When $p \geq 2$ and $1 / 2<\beta \leq 1$, it is easy to see that

$$
-\frac{1}{4} \beta<-\left(\beta-\frac{1}{2}\right) \frac{1}{2} \leq-\left(\beta-\frac{1}{2}\right) \frac{1}{p} .
$$

For a given $\beta$, we can then take $\alpha \in(0,1 / 2)$ sufficiently close to $1 / 2$ such that

$$
-\frac{1}{4} \beta<-\alpha^{2} \beta<-\left(\beta-\frac{1}{2}\right) \frac{1}{p} .
$$

Therefore, our method gives a better estimate of the convergence rate than the EulerMaruyama scheme for $\beta \in[1 / 2,1)$. For $\beta \in(0,1 / 2)$, the convergence of the EulerMaruyama approximation is not known. Furthermore, as we prove later, Theorem 3.8 below implies that the estimated rate in Theorem 3.2 is not sharp when $\sigma$ is smooth. 
Remark 3.7. Giles [5] introduced the Multi-level Monte Carlo method for reducing the computational complexity to achieve a given mean-square-error tolerance level:

$$
E\left[\left(\hat{Y}-E\left[f\left(X_{T}\right)\right]\right)^{2}\right]<\epsilon,
$$

where $\hat{Y}$ is the estimator of $E\left[f\left(X_{T}\right)\right]$ by combination of the Euler-Maruyama method and the Multi-level Monte Carlo method. It is proven in [5] that a better strong rate of convergence can reduce the computational complexity. We therefore expect our discretization scheme to be more effective than the Euler-Maruyama method for the multilevel Monte Carlo method when the diffusion coefficient is irregular. We leave this as work for the future.

We now provide a better estimate of the convergence rate of our scheme when $\sigma$ is smooth. Denote by $\mathcal{L}^{q}$ the class of stochastic processes $\left\{X_{t}\right\}$ and $q \in \mathbb{N}$ such that

$$
E\left[\int_{0}^{t}\left|X_{s}\right|^{q} d s\right]<\infty, \quad 0 \leq t<\infty,
$$

and by $\sigma_{t}, \sigma_{x}, \sigma_{x, x}$ the partial derivatives of $\sigma$ :

$$
\sigma_{t}(t, x):=\frac{\partial \sigma}{\partial t}(t, x), \sigma_{x}(t, x):=\frac{\partial \sigma}{\partial x}(t, x), \sigma_{x x}(t, x):=\frac{\partial^{2} \sigma}{\partial x^{2}}(t, x) .
$$

Theorem 3.8. Suppose that $\sigma:[0, \infty) \times \mathbb{R} \mapsto \mathbb{R}$ belongs to $C^{2,2}$ and satisfies the following conditions in addition to Condition 3.1:

(i) For any $T>0$, there exists a constant $L_{T}>0$ such that

$$
|\sigma(s, x)-\sigma(t, x)| \leq L_{T}|s-t|, \quad \forall x \in \mathbb{R}, \forall s, t \in[0, T] .
$$

(ii) There exists some positive constants $C_{3}, C_{4}$ such that

$$
\left|\sigma_{x x}(t, x)\right|+\left|\sigma_{t}(t, x)\right| \leq C_{3} \exp \left\{C_{4}(t+|x|)\right\}, \quad \forall x \in \mathbb{R}, \forall t \in[0, T] .
$$

Then for all $T>0, \alpha \in(0,1 / 2)$ and $p \geq 1$, there exists some constant $K_{T}>0$ such that

$$
\left\{E\left[\sup _{t \leq T}\left|\xi_{\tau_{n}(t)}^{(n)}-\xi_{\tau(t)}\right|^{p}\right]\right\}^{1 / p} \leq K_{T} n^{-\alpha}
$$

Remark 3.9. Since $\varphi(t)$ satisfies (2.1), by Condition 3.1, $\varphi(t)$ is bounded by $C_{1}^{-2} t$. Then, under the condition (ii) of Theorem 3.8, $\sigma_{t}\left(\varphi(t), \xi_{t}\right), \sigma_{x}\left(\varphi(t), \xi_{t}\right)$ and $\sigma_{x, x}\left(\varphi(t), \xi_{t}\right)$ belong to $\mathcal{L}^{q}$ for any $q \in \mathbb{N}$.

Proof of Theorem 3.8. Under the assumptions of this theorem, (3.7)-(3.12) continue to hold. Therefore, it remains only to estimate the convergence rate of (3.12). More precisely, it remains to prove that for $\alpha \in(0,1 / 2)$ and $T>0$ there exists a constant $K_{T}>0$ such that

$$
\left\{E\left[\sup _{t \leq T^{\prime}}\left|\varphi(t)-\varphi_{n}(t)\right|^{2 p \alpha}\right]\right\}^{1 / 2 p} \leq K_{T} n^{-\alpha}
$$

We denote by $C$ a generic constant which depends on $p, \alpha$, and $T$, and may change line by line. Note that $\xi_{t}=\xi_{0}+b_{t}$, where $b_{t}$ is a standard Brownian motion, and let us write $X_{t}:=\sigma^{-2}\left(\varphi(t), \xi_{t}\right)$. Since $\sigma(t, x)$ belongs to $C^{2,2}, X_{t}$ is a semimartingale and can be written as

$$
X_{t}=X_{0}+M_{t}+B_{t},
$$


where

$$
X_{0}:=\sigma^{-2}\left(0, \xi_{0}\right), \quad M_{t}:=\int_{0}^{t} \gamma_{s} d b_{s}, \quad B_{t}:=\int_{0}^{t} \delta_{s} d s,
$$

and $\left\{\gamma_{t}\right\}$ and $\left\{\delta_{t}\right\}$ are in $\mathcal{L}^{q}$ for any $q \in \mathbb{N}$.

Note that

$$
\begin{aligned}
& \varphi_{n}(t)-\varphi(t) \\
& =\int_{0}^{t} \sigma^{-2}\left(\varphi_{n}\left(\frac{\lfloor n s\rfloor}{n}\right), \xi_{\frac{\lfloor n s\rfloor}{n}}\right) d s-\int_{0}^{t} X_{s} d s \\
& =\int_{0}^{t}\left\{X_{\frac{\lfloor n s\rfloor}{n}}-X_{s}\right\} d s+\int_{0}^{t}\left\{\sigma^{-2}\left(\varphi_{n}\left(\frac{\lfloor n s\rfloor}{n}\right), \xi_{\frac{\lfloor n s\rfloor}{n}}\right)-\sigma^{-2}\left(\varphi\left(\frac{\lfloor n s\rfloor}{n}\right), \xi_{\frac{\lfloor n s\rfloor}{n}}\right)\right\} d s
\end{aligned}
$$

Since $\sigma^{-2}(t, x)$ is locally Lipschitz continuous in $t$ uniformly w.r.t. $x$, for $t \leq T^{\prime}$ we have

$$
\begin{aligned}
E\left[\sup _{s \leq t}\left|\varphi_{n}(s)-\varphi(s)\right|^{2 p \alpha}\right] \leq & C E\left[\sup _{s \leq t}\left|\int_{0}^{s} X_{\frac{\lfloor n u\rfloor}{n}}-X_{u} d u\right|^{2 p \alpha}\right] \\
& +C \int_{0}^{t} E\left[\sup _{u \leq s}\left|\varphi_{n}(u)-\varphi(u)\right|^{2 p \alpha}\right] d s
\end{aligned}
$$

Here we use the fact that there is a positive constant $C$ depending on $2 p \alpha$ such that $|x+y|^{2 p \alpha} \leq C\left\{|x|^{2 p \alpha}+|y|^{2 p \alpha}\right\}$. In fact, when $2 p \alpha \geq 1$ it is clear from the convex property of $|\cdot|^{2 p \alpha}$, and $C=1$ when $0<2 p \alpha<1$ by a simple discussion of the derivative of $|\cdot|^{2 p \alpha}$. Since we can regard the two expectations in the left- and right-hand side of (3.21) as functions of $t$, by Gronwall's lemma, we get

$$
E\left[\sup _{s \leq t}\left|\varphi_{n}(s)-\varphi(s)\right|^{2 p \alpha}\right] \leq C E\left[\sup _{t \leq T^{\prime}}\left|\int_{0}^{t} X_{\frac{\lfloor n s\rfloor}{n}}-X_{s} d s\right|^{2 p \alpha}\right] .
$$

Using by parts formula for $t X_{t}$ and $s X_{s}(s<t)$,

$$
\int_{s}^{t}\left(X_{u}-X_{s}\right) d u=\int_{s}^{t}(t-u) d X_{u}, \quad \text { a.s. }
$$

and letting $t_{i}=i / n, i=0,1, \cdots$, we obtain

$$
\begin{aligned}
E\left[\sup _{t \leq T^{\prime}}\left|\int_{0}^{t} X_{\frac{\lfloor n s\rfloor}{n}}-X_{s} d s\right|^{2 p \alpha}\right] & =E\left[\sup _{t \leq T^{\prime}}\left|\sum_{i=1}^{\lfloor n t\rfloor} \int_{t_{i-1}}^{t_{i}} X_{s}-X_{t_{i-1}} d s+\int_{\frac{\lfloor n t\rfloor}{n}}^{t} X_{s}-X_{\frac{\lfloor n t\rfloor}{n}} d s\right|^{2 p \alpha}\right] \\
& =E\left[\sup _{t \leq T^{\prime}}\left|\sum_{i=1}^{\lfloor n t\rfloor} \int_{t_{i-1}}^{t_{i}}\left(t_{i}-s\right) d X_{s}+\int_{\frac{\lfloor n t\rfloor}{n}}^{t}(t-s) d X_{s}\right|^{2 p \alpha}\right] \\
& =E\left[\sup _{t \leq T^{\prime}}\left|\int_{0}^{\frac{\lfloor n t\rfloor}{n}}\left(\frac{\lfloor n s\rfloor+1}{n}-s\right) d X_{s}+\int_{\frac{\lfloor n t\rfloor}{n}}^{t}(t-s) d X_{s}\right|^{2 p \alpha}\right] .
\end{aligned}
$$

This implies that

$$
\begin{aligned}
E\left[\sup _{t \leq T^{\prime}}\left|\int_{0}^{t} X_{\frac{\lfloor n s\rfloor}{n}}-X_{s} d s\right|^{2 p \alpha}\right] \leq & C E\left[\sup _{t \leq T^{\prime}}\left|\int_{0}^{t}\left(\frac{\lfloor n s\rfloor+1}{n}-s\right) d X_{s}\right|^{2 p \alpha}\right] \\
& +C E\left[\sup _{t \leq T^{\prime}}\left|\int_{0}^{t}\left(\frac{\lfloor n s\rfloor+1}{n} \wedge t-\frac{\lfloor n s\rfloor+1}{n}\right) d X_{s}\right|^{2 p \alpha}\right] .
\end{aligned}
$$


Because of the fact that $\frac{\lfloor n s\rfloor+1}{n} \wedge t-\frac{\lfloor n s\rfloor+1}{n}=0$ for $s<\frac{\lfloor n t\rfloor}{n}$, the definition $X_{s}=$ $\sigma^{-2}\left(\varphi(s), \xi_{s}\right)$ and Condition 3.1, we can estimate the second term in (3.25) as follows.

$$
\begin{aligned}
& E\left[\sup _{t \leq T}\left|\int_{0}^{t}\left(\frac{\lfloor n s\rfloor+1}{n} \wedge t-\frac{\lfloor n s\rfloor+1}{n}\right) d X_{s}\right|^{2 p \alpha}\right] \\
= & E\left[\sup _{t \leq T}\left|\int_{\frac{\lfloor n t\rfloor}{n}}^{t}\left(t-\frac{\lfloor n t\rfloor+1}{n}\right) d X_{s}\right|^{2 p \alpha}\right] \\
= & E\left[\sup _{t \leq T}\left|\left(t-\frac{\lfloor n t\rfloor+1}{n}\right)\left(X_{\frac{\lfloor n t\rfloor}{n}}-X_{t}\right)\right|^{2 p \alpha}\right] \\
\leq & E\left[\frac{1}{n^{2 p \alpha}} \sup _{t \leq T}\left|X_{\frac{\lfloor n t\rfloor}{n}}-X_{t}\right|^{2 p \alpha}\right] \leq \frac{C}{n^{2 p \alpha}}
\end{aligned}
$$

To estimate the first term, we recall the notation (3.17) and obtain

$$
\begin{aligned}
& E\left[\sup _{t \leq T}\left|\int_{0}^{t}\left(\frac{\lfloor n s\rfloor+1}{n}-s\right) d X_{s}\right|^{2 p \alpha}\right] \\
\leq & C\left\{E\left[\sup _{t \leq T}\left|\int_{0}^{t}\left(\frac{\lfloor n s\rfloor+1}{n}-s\right) \delta_{s} d s\right|^{2 p \alpha}\right]+E\left[\sup _{t \leq T}\left|\int_{0}^{t}\left(\frac{\lfloor n s\rfloor+1}{n}-s\right) \gamma_{s} d b_{s}\right|^{2 p \alpha}\right]\right\}
\end{aligned}
$$

Recalling Remark 3.9, the $\mathcal{L}^{2 p \alpha \vee 1}$ property of $\delta_{t}$ implies that

$$
E\left[\sup _{t \leq T}\left|\int_{0}^{t}\left(\frac{\lfloor n s\rfloor+1}{n}-s\right) \delta_{s} d s\right|^{2 p \alpha}\right] \leq \frac{1}{n^{2 p \alpha}} E\left[\left|\int_{0}^{T}\right| \delta_{s}|d s|^{2 p \alpha}\right] \leq \frac{C}{n^{2 p \alpha}} .
$$

Here we have used the fact that

$$
E\left[\left|\int_{0}^{T}\right| \delta_{s}|d s|^{2 p \alpha}\right] \leq T^{2 p \alpha-1} E\left[\int_{0}^{T}\left|\delta_{s}\right|^{2 p \alpha} d s\right]<\infty
$$

if $2 p \alpha \geq 1$ and

$$
E\left[\left|\int_{0}^{T}\right| \delta_{s}|d s|^{2 p \alpha}\right] \leq E\left[\int_{0}^{T}\left|\delta_{s}\right| d s\right]^{2 p \alpha}<\infty
$$

if $2 p \alpha<1$.

By using the Burkholder-Davis-Gundy inequality and the $\mathcal{L}^{2 p \alpha}$ property of $\gamma_{t}$, we obtain the following for the second term of (3.27):

$$
E\left[\sup _{t \leq T}\left|\int_{0}^{t}\left(\frac{\lfloor n s\rfloor+1}{n}-s\right) \gamma_{s} d b_{s}\right|^{2 p \alpha}\right] \leq C E\left[\left|\int_{0}^{T}\right| \frac{\lfloor n s\rfloor+1}{n}-\left.\left.s\right|^{2} \gamma_{s}^{2} d s\right|^{p \alpha}\right] \leq \frac{C}{n^{2 p \alpha}} .
$$

From (3.25) and (3.26)-(3.29), it follows that

$$
E\left[\sup _{t \leq T^{\prime}}\left|\int_{0}^{t} X_{\frac{\lfloor n s\rfloor}{n}}-X_{s} d s\right|^{2 p \alpha}\right] \leq \frac{C}{n^{2 p \alpha}}
$$

which concludes the proof. 


\section{Numerical example}

In this section, we provide some numerical examples for the cases where the diffusion coefficients of (1.1) are $1 / 3,1 / 2$ and $3 / 4$-Hölder continuous. As a specific example of this kind of coefficient, we choose the Weierstrass function

$$
\sum_{n=0}^{\infty} a^{n} \cos \left(b^{n} \pi x\right)
$$

where $0<a<1, b>1$ and $a b>1$. According to [8], this function is $(-\log a / \log b)$-Hölder continuous. Therefore, by choosing parameters $b=3$ and $a=3^{-1 / 3}, 3^{-1 / 2}, 3^{-3 / 4}$, we can construct $1 / 3,1 / 2,3 / 4$-Hölder continuous functions respectively. For the diffusion coefficient to satisfy Condition 3.1 , we set

$$
\sigma(t, x)=\sigma(x)=\varepsilon+\frac{a}{1-a}+\sum_{n=0}^{\infty} a^{n} \cos \left(b^{n} \pi x\right),
$$

with some positive constant $\varepsilon>0$. Let us consider the SDE (1.1) with diffusion coefficient $\sigma$ as (4.2). Since the infinite sum (4.1) is not implementable, we approximate it by the sum of the first 1000 terms. We now compare the numerical simulation by root square approximation error

$$
\left\{E\left[\left|X_{T}-Y_{T}^{(n)}\right|^{2}\right]\right\}^{1 / 2}
$$

where $Y_{T}^{(n)}$ is discretization of solution to the SDE (1.1) by the Euler-Maruyama method or our method. The integer $n \in \mathbb{N}$ is regarded as the same variable as $n$ in (2.3) when we consider the rate of convergence for the new method. When we consider the rate for the Euler-Maruyama approximation, $n$ is the same variable as $n$ in (1.2). We let $T=1$, $X_{0}=0$, and $n \in\left\{2^{3}, 2^{4}, \cdots, 2^{9}\right\}$. Taking $\left\{Y_{t}^{(10)}\right\}_{t \leq T}$ as the exact solution of the SDE (1.1), we consider the strong approximation error (4.3). Figure 1 shows the error convergence of both methods.
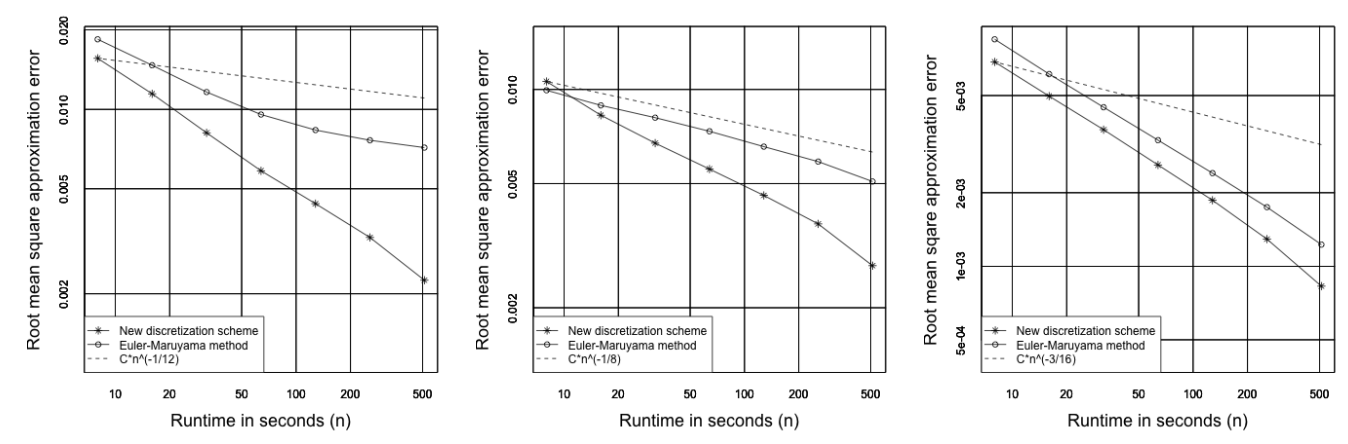

Figure 1: Root mean square approximation error of the Euler-Maruyama method and our new discretization method when $n \in\left\{2^{3}, 2^{4}, \cdots, 2^{9}\right\}$, with sample size $10^{5}$. The above figures illustrate, from left to right, the cases of $1 / 3,1 / 2,3 / 4$-Hölder continuous diffusion coefficient. The broken line in the figure on the left is $C n^{-1 / 12}$, where $C$ is a positive constant such that $C\left(2^{3}\right)^{-1 / 12}$ equals the error of our discretization method with $n=2^{3}$. By this line, we attempt to visualize the theoretical decay in the results of Theorem 3.2 when the Hölder index $\beta$ is $1 / 3$. The same applies to the remaining figures.

In all cases, the proposed method appears to achieve a faster rate of convergence than guaranteed by Theorem 3.2. When the Hölder exponent is $1 / 3$, the numerical 
approximation error of our method appears to decay linearly in log-log graph, while the error of the Euler-Maruyama method appears to decay logarithmically. Here we remark the fact that the convergence of Euler-Maruyama method is not guaranteed when the Hölder index is less than $1 / 2$. When the Hölder exponent is $1 / 2$, we note that the numerical result of the Euler-Maruyama method suggests a faster rate than the estimate (1.3) of logarithmic convergence. Nevertheless, we observe that the speed of convergence for the new method is faster than the Euler-Maruyama method. When the Hölder index is 3/4, there is no apparent difference in the speed of convergence, but the numerical approximation error of both methods seem to decay linearly in log-log graph, as the theoretical results suggest.

\section{References}

[1] Barlow, M. T.: One-dimensional stochastic differential equations with no strong solution. J. London Math. Soc. (2) 26 (1982), no. 2, 335-347. MR-0675177

[2] Brox, T.: A one-dimensional diffusion process in a Wiener medium. Ann. Probab. 14 (1986), no. 4, 1206-1218. MR-0866343

[3] Bru, B. and Yor, M.: Comments on the life and mathematical legacy of Wolfgang Doeblin. Finance Stoch. 6 (2002), no. 1, 3-47. MR-1885582

[4] Engelbert, H. J. and Schmidt, W.: On the behaviour of certain functionals of the Wiener process and applications to stochastic differential equations. Stochastic differential systems (Visegrád, 1980), pp. 47-55, Lecture Notes in Control and Information Sci., 36, Springer, Berlin-New York, 1981. MR-0653645

[5] Giles, M. B.: Multilevel Monte Carlo path simulation. Oper. Res. 56 (2008), no. 3, 607-617. MR-2436856

[6] Grigorian, A.: Ordinary differential equation (2009). https://www.math.uni-bielefeld.de/ $\sim$ grigor/odelec2009.pdf

[7] Gyöngy, I. and Rásonyi, M.: A note on Euler approximations for SDEs with Hölder continuous diffusion coefficients. Stochastic Process. Appl. 121 (2011), no. 10, 2189-2200. MR-2822773

[8] Hardy, G. H.: Weierstrass's non-differentiable function. Trans. Amer. Math. Soc. 17 (1916), no. 3, 301-325. MR-1501044

[9] Hu, Y., Lê, K. and Mytnik, L.: Stochastic differential equation for Brox diffusion. Stochastic Process. Appl. 127 (2017), no. 7, 2281-2315. MR-3652414

[10] Ikeda, N. and Watanabe, S.: Stochastic differential equations and diffusion processes. Second edition. North-Holland Mathematical Library, 24. North-Holland Publishing Co., Amsterdam; Kodansha, Ltd., Tokyo (1989). xvi+555 pp. ISBN: 0-444-87378-3. MR-1011252

[11] Karatzas, I. and Shreve, S. E.: Brownian motion and stochastic calculus. Second edition. Graduate Texts in Mathematics, 113. Springer-Verlag, New York (1991). xxiv+470 pp. ISBN: 0-387-97655-8. MR-1121940

[12] Kloeden, P. E. and Platen, E.: Numerical solution of stochastic differential equations. Applications of Mathematics (New York), 23. Springer-Verlag, Berlin (1992). xxxvi+632 pp. ISBN: 3-540-54062-8. MR-1214374

[13] Revuz, D. and Yor, M.: Continuous martingales and Brownian motion. Third edition. Grundlehren der Mathematischen Wissenschaften [Fundamental Principles of Mathematical Sciences], 293. Springer-Verlag, Berlin, (1999). xiv+602 pp. ISBN: 3-540-64325-7. MR1725357

[14] Taguchi, D.: Numerical analysis for stochastic differential equations with irregular coefficients. (Unpublished doctoral dissertation). Ritsumeikan University (2017). https://ci.nii.ac. jp/naid/500001044256 